\title{
Ökonomie erhebt sich über Medizin
}

\author{
Michel Romanens ${ }^{a}$, Ansgar Adams ${ }^{b}$, Franz Ackermann ${ }^{c}$, Bernhard Hofmeier ${ }^{d}$, Flavian Kurth ${ }^{e}$, Walter Warmuth \\ ${ }^{a}$ Dr. med., Vascular Risk Foundation (VARIFO); ${ }^{b}$ Dr. med., Arzt für Arbeitsmedizin, B.A.D Gesundheitsvorsorge und Sicherheitstechnik GmbH; ${ }^{\circ}$ Dr. med., \\ Vascular Risk Foundation (VARIFO); ${ }^{d}$ Dr. med., Fairfond Stiftung für Fairness im Gesundheitswesen; ${ }^{e}$ Flavian Kurth, Sekretär, Verein Ethik und Medizin \\ Schweiz (VEMS); ${ }^{\dagger}$ Dr. rer. nat. habil., Gesundheitsforen Leipzig
}

Ob die Mathematik eine Natur- oder eine Geisteswissenschaft ist, wird kontrovers diskutiert. Anwendung findet sie sowohl in den Natur-, als auch in den Geisteswissenschaften, wobei gilt: "Alle mathematischen Modelle sind falsch, manche sind nützlich» [1]*. Für diese «Nützlichkeit» gibt es bei naturwissenschaftlichen Anwendungen ein unkorrumpierbares Regulativ: die physikalische Realität und ihre Naturgesetze. Es ist hier empirisch überprüfbar, ob es sich um eine "Abbildung» einer gegebenen naturwissenschaftlichen Theorie handelt oder nicht. In den Geisteswissenschaften wird hervorgehoben, dass es sich bei den von ihnen verwandten Modellen nicht um Modelle im mathematischen Sinne handelt [2]. Nach den Vorstellungen von «Autoren» werden «Theorienmodelle» generiert. In der Realität werden diese Theorienmodelle unter Annahmen auf ihre «Passfähigkeit» hin validiert, um sie gegebenenfalls zu revidieren. Die Validierung erfolgt mit mathematischen Bewertungen, ein natürliches Regulativ indes fehlt den Geisteswissenschaften. Eine wichtige Rolle spielen deshalb die Regulative durch Politik, Ethik und Recht. Sie sollten verhindern, dass falsche Annahmen und falsche Modelle in die Realität hineinwirken und sie unter Umständen verstören. Die Mathematik kann dabei helfen, indem sie mit statistisch-mathematischen Instrumenten die Bewertungen der Wirkungen untersucht, um die tatsächlichen Sachverhalte aufzuzeigen. Diese Aufgabe nimmt sie im Spannungsfeld von Ökonomie und Medizin zu wenig wahr.

\section{Der SMB-Bericht als alarmierendes Beispiel}

Das Swiss Medical Board (SMB) ist ein Gremium, das in Health Technology Assessments HTA das Kosten-Nutzen-Verhältnis medizinischer Behandlungen untersucht [3]. Wir haben bei den HTAs einerseits die naturwissenschaftlich arbeitende Medizin, deren Erkenntnisse und Fortschritte aus der Aufarbeitung konkreter Einzelfälle resultieren, und andererseits die Gesundheitsökonomie, welche die Sorge um die Bezahlbarkeit dieser Fortschritte dazu bringt, die Erkenntnisse der Medizin vermehrt in Frage zu stellen. Dies tut sie als Geisteswissenschaft mit Annahmen und Modellen, die dann mathematisch zu validieren sind. Als Gremium mit Einsitz der FMH (Medizin), der Gesundheitsdirektorenkonferenz GDK (Politik) und der SAMW (Ethik) und einer Rechtsprofessorin verfügt das SMB grundsätzlich über die internen Regulative zur Erarbeitung verantwortbarer HTA-Berichte. Umso mehr erstaunt das Resultat [4], das Gegenstand dieses Artikels ist: ein Bericht, der entgegen allen nationalen und internationalen Empfehlungen der Fachgesellschaften den äusserst restriktiven Einsatz von Statinen in der Primärprävention kardiovaskulärer Krankheiten empfiehlt. Diese Empfehlung fusst auf mathematischen Berechnungen, die in ihrer Komplexität beeindrucken und vielleicht auch abschre-

Der methodische Fehler des SMB-Statinberichts liegt in einer falschen Risikobeurteilung.

cken. Der Verein Ethik und Medizin Schweiz (VEMS) hat sich davon nicht blenden lassen und nachgerechnet [5] Im Ergebnis zeigt sich, dass die Berechnungen des SMB gravierende Fehler enthalten, was der Grund dafür sein dürfte, dass das SMB nun darüber nachdenkt, seinen Statinbericht zurückzuziehen, um seine wissenschaftliche Reputation zu wahren. Wenn es zu diesem Schritt nun aber der Bemühungen des VEMS bedurfte, dann zeigt das doch, dass die internen Regulative des SMB offensichtlich nicht funktionieren.

\section{Der grundsätzliche methodische Mangel des SMB-Berichts}

Der methodische Fehler des SMB-Statinberichts liegt in einer falschen Risikobeurteilung. Die Frage, ab welchen Werten ein Patient als Risikopatient für Herz-KreislaufKrankheiten gilt, ist von verschiedenen nationalen und internationalen Gremien untersucht worden, die leicht variierende Richtwerte angeben [6]. Das SMB hat einen Wert angenommen, der erheblich über den Werten aller internationalen Fachgesellschaften liegt, um dann für eine spezielle, in der medizinischen Praxis kaum erheb- 
liche Patientengruppe "nachzuweisen", dass erst ab diesem Wert eine Behandlung mit Statinen nötig sei. Daraus wurde eine allgemeine Empfehlung für alle Patientengruppen abgleitet, was eine Verzerrung der Tatsachen ist und wissenschaftlichen Regeln widerspricht [7].

\section{Mathematische Mängel des SMB-Berichts}

Das SMB arbeitet in seinen Berechnungen mit dem QALY-Konzept [8], das es ermöglicht, den medizinischen Nutzen in einer Zahl zu fassen. Denn um ein KostenNutzen-Verhältnis aufstellen zu können, muss für beides, für die Kosten und für den Nutzen, ein Zahlenwert errechenbar sein. Das QALY-Konzept tut dies, indem für den medizinischen Nutzen ein qualitätsadjustierter Wert pro gewonnenem Lebensjahr gesetzt wird. Wie willkürlich dieser Wert gesetzt werden kann, zeigt nun aber der SMB-Statinbericht exemplarisch. Das SMB basiert seine Berechnungen auf der Annahme, dass bei 1000 gesunden Personen mit Statinen zwei tödliche Herzinfarkte in fünf Jahren vermieden werden. Diesem seien neun nicht tödliche, aber vermeidbare Herzinfarkte zuzuordnen (also 4,5 nicht tödliche auf einen tödlichen Herzinfarkt). Die Wirksamkeit der Statine wird mit einer relativen Risikoreduktion von $22 \%$ angegeben. Im Ergebnis kostet bei diesem Szenario ein gewonnenes Lebensjahr in guter Gesundheit 210000 CHF pro QALY, unbestreitbar ein zu hoher Preis. Falls die Berechnungen des SMB stimmen. Das tun sie aber nicht, denn die Frage lautet hier richtig: Bei welchem Risiko lassen sich zwei tödliche und neun nicht tödliche Herzinfarkte mit Statinen vermeiden? Bei einer relativen Risikoreduktion von $22 \%$ braucht es also neun tödliche und 41 nicht tödliche Herzinfarkte, um die oben erwähnten zwei tödlichen und neun nicht tödlichen Herzinfarkte zu vermeiden. Neun tödliche Herzinfarkte bei 1000 Personen entsprechen einem ESC-Risiko von 0,9\% in fünf Jahren. Da die relative Risikoreduktion auch nach mehr als fünf Jahren unverändert erhalten bleibt, entspricht das Rechenbeispiel des SMB einem 10-JahresESC-Risiko von $2 \times 0,9 \%=1,8 \%$. Das SMB schreibt aber, dass die Kosten von 210000 CHF pro QALY für ein Risiko von $5 \%$ in 10 Jahren gelten würden. Tatsächlich wäre dies korrekt, wenn man die nicht tödlichen Herzinfarkte einberechnete. Dann resultieren neun tödliche + 41 nicht tödliche $=50$ Herzinfarkte in fünf Jahren, woraus sich ein Risiko von 5\% (50/1000) ergibt. Auf 10 Jahre hochgerechnet resultiert so ein Risiko von $10 \%$. Womit wir bei den Kostenwirksamkeitsergebnissen des ersten SMB-Statinberichts sind, welche im zweiten, überarbeiteten Bericht auf 7,5\% herunterkorrigiert wurden, aber auch dort noch immer falsch sind. In beiden Berichten verwechselt das SMB offensichtlich das ESC-Sterberisiko mit dem Risiko anderer Rechner wie PROCAM, FRAMINGHAM usw., bei denen auch nicht tödliche Herzinfarkte berücksichtigt werden. Im zweiten SMB-Bericht wird nun das ESC-Risiko wie erwähnt von 10\% auf 7,5\% als untere Grenze für die Indikation einer Statinbehandlung bei Gesunden heruntergesetzt, angeblich mit Kosten von 160000 CHF pro QALY. Dies ohne eine nachvollziehbare Erklärung, ohne Hinweis auf eine begründende Formel oder eine Methode der Herleitung dieser Zahl. Die korrekten Kosten pro QALY, gerechnet mit den Annahmen des SMB: Für ein ESC-Risiko von 2,5\% in fünf Jahren ergeben sich 53110 CHF pro QALY. Bei 10 Jahren wird das Risiko auf 5\% verdoppelt, und entsprechend dem Modell wird der QALY-Wert vervierfacht, wie die Berechnungen des VEMS im wissenschaftlichen Austausch mit den Autoren und beigezogenen Spezialisten ergeben haben. Ergebnis folglich: $17081 \mathrm{CHF}$ pro QALY. Für ein ESC-Risiko von 3,75\% in fünf Jahren ergeben sich 23126 CHF pro QALY, entsprechend in 10 Jahren mit einem ESC-Risiko von 7,5\% 2089 CHF pro QALY. Für die vom SMB angegebenen ESC-Sterberisiken zeigt das Beispielmodell also sehr hohe, tatsächlich kaum akzeptable Kosten pro QALY. Nur gelten diese Kosten eben für ein Risiko von 0,9\% und nicht für ein Risiko von 3,75\% in fünf Jahren, oder, auf 10 Jahre gerechnet, von $7,5 \%$. Dass ein Konzept Zahlen im Bereich von 210000 CHF bis 2089 CHF pro QALY hervorbringen und plausibilisieren kann, macht nicht eben einen wissenschaftlichen Eindruck.

\section{Die juristischen und gesellschaftlichen Implikationen des SMB-Berichts}

Vor oben aufbereitetem Hintergrund sind die juristischen Implikationen des SMB-Statinberichts nicht haltbar. Das SMB postuliert: "Aus juristischer Sicht kann man sich hier sogar fragen, ob der Wirkungsnachweis für eine vorwiegend männliche und deutlich jüngere Population ... nicht sogar als Nachweis von etwas anderem, als juristisches ‘aliud betrachtet werden könnte.» Hier werden die Dinge nun komplett auf den Kopf gestellt, indem die Rechtmässigkeit empirisch erwiesener Tatsachen der klinischen Praxis aufgrund von Berechnungen in Frage gestellt wird, die reine Zahlenspiele einer aus der medizinischen Praxis ausgelagerten und ohne Bezug zu ihr rechnenden Ökonomie sind. Das Recht nimmt seine Aufgabe als Regulativ nicht wahr, wird vielmehr zum Diener von Annahmen im Kampf gegen Tatsachen. Fakt ist: Das Problem der Atherosklerose ist erwiesenermassen in der Bevölkerung prävalent. Das SMB diskutiert dieses aber nur im Zusammenhang mit der Jupiterstudie und postuliert, Gesunde 
seien gesund, was impliziert, dass auch kranke Arterien gesund seien oder jedenfalls nicht behandlungsbedürftig. Damit wird eine volksgesundheitliche Dimension tangiert, und es stellt sich doch die Frage, wer in unserem Gesundheitswesen die Definitionshoheit darüber haben soll, was gesund sei und was krank, die Ökonomie oder die Medizin. Letztere stellt fest: Mit seinem Rechenbeispiel «belegt» das SMB sein Postulat, denn bei Personen mit einem ESC-Risiko von 0,9\% in 5 Jahren kann man davon ausgehen, dass sie relativ selten eine relevante Atherosklerose aufweisen. Die Vascular Risk Foundation (VARIFO) hat dies bei 1601 Personen in der Schweiz überprüft. Eine relevante Atherosklerose, bestimmt mit der TPA-Methode (TPA >80 $\mathrm{mm}^{2}$ ), zeigte sich bei $13 \%$ der Personen mit einem ESC-Risiko $<0,9 \%$. Bei einem ESC-Risiko von $\geq 0,9 \%$ haben $76 \%$ eine Atherosklerose mit mässig hohem oder hohem Risiko und bei 18\% eine Atherosklerose mit hohem Risiko. Damit verkennt das SMB die Tatsache, dass Personen bereits mit einem ESC-Risiko von grösser als 0,9\% in 10 Jahren in 91\% eine relevante Atherosklerose und in 35\% eine schwere Atherosklerose aufweisen. Die VARIFO hat deshalb in der Schweizerischen Ärztezeitung [9] das SMB gefragt, ob die Lösung darin bestehen könnte, die mittels Bildgebung erfasste Atherosklerose, also die Krankheit Atherosklerose, als eine Form der "manifesten» Atherosklerose zu sehen. Die Antwort von Prof. Dr. med. Christoph A. Meier lautete, man würde dabei Karotisstenosen entdecken, was zu einer Überbehandlung der Karotisstenosen führen würde. Auch hier verkennt das SMB allerdings die Fakten: bei asymptomatischen, "gesunden» Personen erfolgt mit der TPA-Methode nie eine Suche nach Karotisstenosen. Diese sind zudem bei relativ jungen Personen um 50-65, die besonders für die Prävention mit Statinen interessieren, kaum je anzutreffen. Wenn die Gesundheitsökonomie so komplett an der medizinischen Praxis vorbeidenkt und -rechnet und dieser qua Recht seine falsche Sicht aufzwingt, dann erfüllen beide ihren Gesellschaftsauftrag nicht.

\section{Die Implikationen des SMB-Berichts auf die medizinische Praxis}

Eine kleinere Gefahr des SMB-Statinberichts besteht darin, dass Ärzte der Empfehlung des SMB folgen und ihre Patienten falsch beraten, was insbesondere bei Hausärzten und bei gegebenem Druck durch die Krankenkassen eine Gefahr darstellt, während Kardiologen die Mangelhaftigkeit des SMB-Berichts nicht nur leicht erkennen können, sondern Krankenkassen gegenüber aufgrund ihres Wissensvorsprungs auch besser gegen diese falschen Empfehlungen zu argumentieren vermö- gen. Da Statine aufgrund ihrer nicht unmittelbar spürbaren Wirkung eine eher schlechte Compliance haben (kooperatives Verhalten des Patienten), besteht aber die grössere Gefahr des Berichts darin, dass Patienten, bei denen eine medikamentöse Behandlung eindeutig angezeigt ist und eine Nichtbehandlung tödliche Folgen haben kann, ihre Medikamente, ermutigt durch die Empfehlung des SMB, nicht mehr einnehmen. Dass diese Gefahr real ist, zeigt ein Blick auf die Datenbank der VARIFO. Bei 1601 gesunden Schweizer Personen im mittleren Alter von $55 \pm 7$ Jahren besteht gemäss ESCRichtlinien eine Indikation für die medikamentöse LDLSenkung bei 642 (40\%). Würde man erst ab einem ESCRisiko von 7,5\% oder mehr die Indikation für eine Statinbehandlung stellen dürfen, wie dies das SMB in seinen Empfehlungen vorschlägt, dann würde nur eine statt 642 Personen ein Statin erhalten. Dies bedeutet auch, dass es keinen Sinn mehr ergäbe, das Cholesterin überhaupt zu bestimmen. Zur Überprüfung dieser Zahlen haben wir eine deutsche Kohorte mit 2633 gesunden Personen im mittleren Alter von $49 \pm 7$ Jahren eingesehen. Diese hat eine Statinindikation bei 1105 (42\%). Bei einem ESC-Risiko von 7,5\% oder mehr würde in Deutschland gar niemand mehr mit einem Statin behandelt werden $[10]$.

\section{Fazit}

Der SMB-Statinbericht zeigt exemplarisch eine gefährliche Tendenz: Die Annahmen und Modelle der Gesundheitsökonomie werden nicht mehr anhand der Realität validiert und gegebenenfalls revidiert, sondern dieser übergestülpt. Die Regulative spielen nicht, ökonomische Annahmen bekommen vermehrt den Charakter von Dogmen, die nicht mehr hinterfragt werden, vergleichbar mit religiösen Dogmen. Die Geisteswissenschaft Ökonomie siegt zusehends über die Naturwissenschaft Medizin, quasireligiöse Annahmen gelten mehr als fundiert erarbeitete Evidenz der Tatsachen. Dem muss sich die Medizin widersetzen, denn das ist ihr Auftrag im Interesse der Patienten und der Gesellschaft. Hier sind neben der SAMW und der GDK vor allem auch die FMH und die Fachgesellschaften gefordert, endlich entschieden Gegenpositionen zum grundsätzlichen Misstrauen der Gesundheitsökonomie gegenüber der Medizin einzunehmen. Denn die hier aufbereitete Sachlage spricht doch eher dafür, dieser Art, Gesundheitsökonomie zu betreiben, einer Zahlen-, keiner Werteökonomie, mit Misstrauen zu begegnen. Im Gegensatz zur Medizin ist diese den Beweis der Effizienz ihrer Methoden jedenfalls noch schuldig. Ihr destruktives Potential indes ist evident. 


\section{Referenzen}

1 Sachs L. Statistische Methoden. Berlin: Springer-Verlag; 1993. S. 146 .

2 Widmer T, De Rocchi T. Evaluation - Grundlagen, Ansätze und Anwendungen. Zürich/Chur: Rüegger Verlag; 2012.

3 www.medical-board.ch

4 www.medical-board.ch/fileadmin/docs/public/mb/ Fachberichte/2014-07-21 Bericht Statine Final Anpassung.pdf

5 www.varifo.ch/QALYVarifo.xlsx

6 www.varifo.ch/Romanens082014HP.ppt

7 www.public-health.ch/logicio/client/publichealth/file/ EGEP_en.pdf

8 www.medicine.ox.ac.uk/bandolier/painres/download/whatis/ qaly.pdf

9 www.saez.ch/docs/saez/2014/47/de/saez-03013.pdf

10 Medical Costs per QALY of Statins Using the Swiss Medical Board (SMB) assumptions: Observed Effects in Two Large Primary Prevention Cohorts from Germany and Switzerland. Romanens $M$ Ackermann F, Szucs T, Sudano I, Ansgar Adams. Oral presentation Abstr. 94, SGIM Jahresversammlung 2015. Basel 20.-22.5.2015. 